\title{
Intrinsic Arteriovenous Malformation Embedded in the Trigeminal Nerve of a Patient With Trigeminal Neuralgia -Case Report-
}

\author{
Shinya SUMIOKA, ${ }^{1}$ Akinori KONDO, ${ }^{1}$ \\ Hideki TANABE, ${ }^{1}$ and Soichiro YASUDA $^{1}$ \\ ${ }^{1}$ Department of Neurosurgery, Brain, Spine and Neurology Center, \\ Shiroyama Hospital, Habikino, Osaka
}

\begin{abstract}
A 66-year-old man presented with typical right trigeminal neuralgia. Neuroimaging showed a small arteriovenous malformation (AVM) in the right cerebellopontine angle. Suboccipital craniotomy verified that the AVM was almost completely embedded in the root entry zone of the trigeminal nerve and the nerve axis was tilted infero-posteriorly. The patient obtained complete pain relief without sequelae after surgery by transposition of the superior cerebellar artery and correction of the tilted nerve axis. The nidus of the unresected AVM was obliterated by gamma knife radiosurgery.
\end{abstract}

Key words: arteriovenous malformation, microvascular decompression, radiosurgery, trigeminal neuralgia

\section{Introduction}

Trigeminal neuralgia (TN) is mainly caused by arterial compression at the root entry zone (REZ) of the trigeminal nerve and is occasionally caused by a tumor or thickened arachnoid membrane tilting the axis of the nerve. $\mathrm{TN}$ is also caused by the nidus or feeding artery of an arteriovenous malformation (AVM). Several cases of TN were related to a small AVM buried within the trigeminal nerve. ${ }^{6,10,14,17)}$ We present a very rare case of typical TN caused by a small AVM buried within the trigeminal nerve, and describe the preoperative neuroimaging and surgery findings.

\section{Case Report}

A 66-year-old man had suffered severe typical TN for approximately 2 years in the third division of the right trigeminal nerve. The pain gradually became aggravated and refractory. Magnetic resonance (MR) imaging with contrast medium revealed a small enhanced lesion in the right cerebellopontine angle (CPA) and MR cisternography revealed a small flow-void lesion within the right trigeminal nerve (Fig. 1). Vertebral angiography showed a small AVM with a nidus of approximately $5 \mathrm{~mm}$ in diameter around the REZ of the trigeminal nerve (Fig. 2 ). The patient preferred to receive direct surgical treatment for immediate pain relief.

Received June 28, 2010; Accepted February 7, 2011

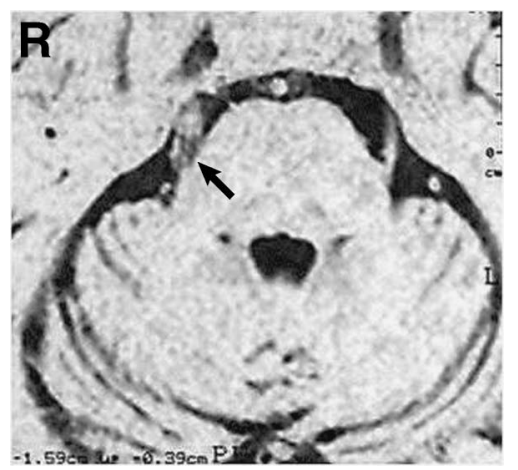

Fig. 1 Magnetic resonance cisternogram revealing a small flow void sign at the proximal site of the right trigeminal nerve (arrow).

The cisternal part of the trigeminal nerve was explored via a right suboccipital craniotomy. One of the superior petrosal veins appeared reddish and a small, red wormlike AVM was found. The nidus of AVM was almost completely embedded in the proximal part of the sensory root of the trigeminal nerve, and the axis of the nerve was tilted infero-posteriorly (Fig. 3A). A small branch of the right anterior inferior cerebellar artery (AICA), which seemed to be a feeder of the AVM, pierced the posterolateral aspect of the pons near the REZ of the nerve. The right superior cerebellar artery (SCA) compressed the nerve at the supero-anterior portion of the REZ of the nerve (Fig. 3B). 


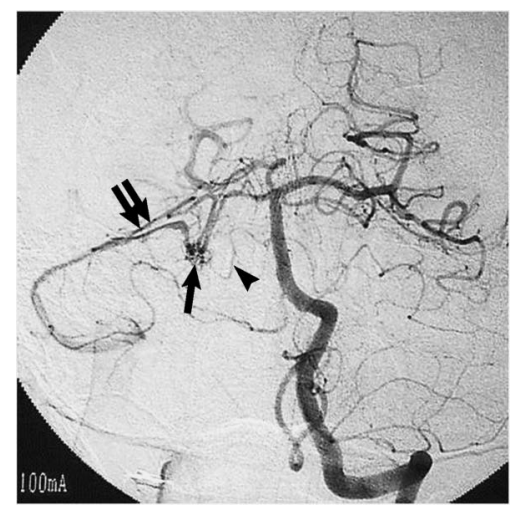

Fig. 2 Left vertebral angiogram showing a small arteriovenous malformation (arrow) for which the feeder is an anterior inferior cerebellar artery (arrowhead) and the drainer is a petrosal vein (double arrow).
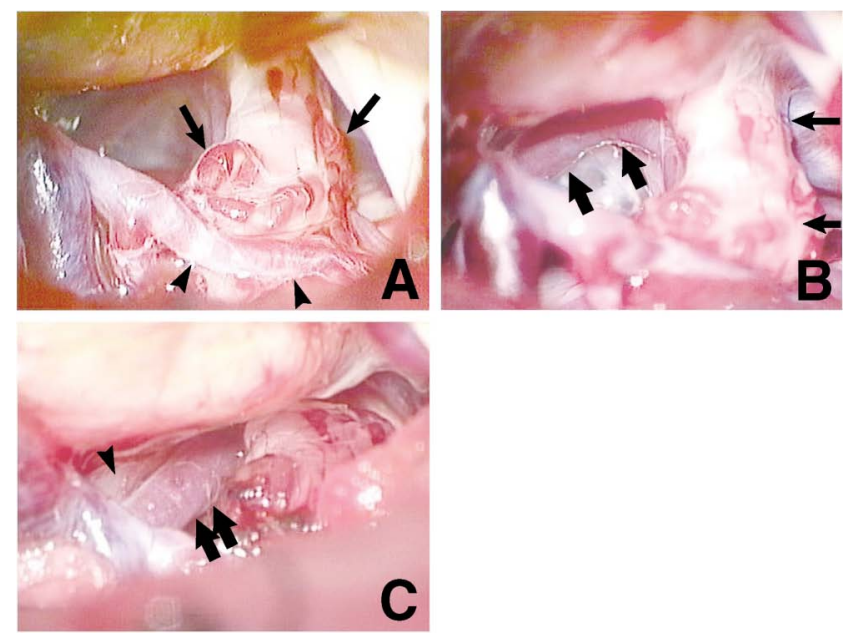

Fig. 3 Intraoperative photographs. A: A small, red wormlike arteriovenous malformation (AVM) was entangled and embedded at the proximal part of the sensory root of the right trigeminal nerve (arrows). The petrosal vein, which was a drainer of the AVM, appeared reddish (arrowheads). B: The right trigeminal nerve (arrows) was compressed by the right superior cerebellar artery (SCA) (thick arrows) at the supero-anterior portion of the root entry zone of the nerve. C: The right SCA (thick arrows) was transpositioned and fixed to the tentorium (arrowhead) with fibrin glue.

This offending artery was successfully transpositioned and fixed to the tentorium with fibrin glue (Fig. 3C). Nidus resection was not attempted for fear of damaging the nerve tissue. Instead, the part of nidus which protruded onto the nerve surface was carefully coagulated to correct the distortion of the nerve axis. After surgery, the patient had complete TN relief without sequelae. One month after surgery, the patient underwent gamma knife radiosurgery to treat the remaining unresected nidus of the AVM. Eighteen months after radiotherapy, MR imaging could not visualize the nidus of the AVM (Fig. 4).

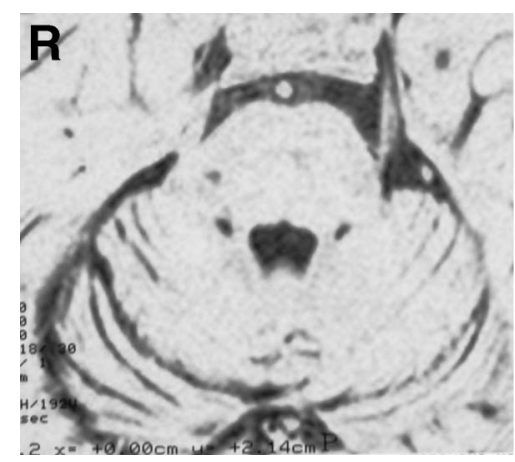

Fig. 4 Magnetic resonance cisternogram at 18 months after radiosurgery showing no nidus of the arteriovenous malformation.

\section{Discussion}

Infratentorial AVMs account for $5 \%$ to $25 \%$ of all intracranial AVMs. ${ }^{2,4,11)}$ AVMs in the brainstem account for $12.5 \%$ to $20 \%$ of infratentorial AVMs. ${ }^{2,4,14)}$ AVM-related $\mathrm{TN}$ is found in approximately $0 \%$ to $1.5 \%$ of patients who have undergone surgery for TN. ${ }^{1,6,8)}$ However, TN resulting from a small intrinsic AVM buried in the trigeminal nerve is extremely rare, with only a few reports of such cases. Five TN cases were caused by a micro-AVM of the trigeminal nerve with the nidus located predominantly subpial and within the trigeminal REZ and the adjacent pons. ${ }^{6}$ The nidus was the cause of TN except in one case, in which conflicting SCA was the cause of TN. Seven cases of cryptic angioma around the nerve root presented as $\mathrm{TN} .{ }^{17)}$ The trigeminal nerve root was encased in a tangle of abnormal vessels, and neither the SCA nor the AICA appeared to compress the nerve root, but whether the vascular malformation was buried in the nerve was not clearly established. The first clear case of an intrinsic AVM in the trigeminal nerve manifesting as TN was detected by MR imaging and angiography. ${ }^{10)}$ The nerve was simply decompressed from the offending SCA, which was believed to be the cause of the TN. An interesting case of intrinsic AVM in the trigeminal nerve was clearly identified within the sensory root, but the patient did not experience any facial pain, and no artery compression on the nerve was observed. ${ }^{14)}$

These previous reports do not establish whether a small AVM within the REZ of the nerve could be the only cause of TN. Most cases of TN are elicited by vascular compression at the REZ of the trigeminal nerve. ${ }^{3,7,9)}$ In our series of 684 patients with TN treated surgically between 1975 and 2005 , approximately $90 \%$ of cases of TN were elicited by compression from a conflicting artery. In the remaining $10 \%$ of cases, TN apparently occurred without any vascular involvement. ${ }^{8,11)}$ In such cases, TN is caused by tilted nerve axis due to a CPA tumor or thickened arachnoid membrane around the nerve. Even a small AVM buried in the trigeminal nerve may start to compress the REZ and distort the axis of the nerve root as the AVM increases in size. ${ }^{16)}$ In our present case, the main cause of TN was se- 
vere compression of the REZ by the SCA, but distortion of the nerve axis by the small AVM buried within the nerve might have been another contributing factor. Since the patient had no post-surgical sequelae, coagulation of part of the nidus did not have much effect on the nerve function.

The goal of treating TN in a patient with an AVM buried in the trigeminal nerve is to relieve pain and to lessen the risk of hemorrhage, while preserving normal nerve function. Successful surgical resection of an intrinsic AVM in the trigeminal nerve without sequelae is quite difficult. Most previous patients have suffered moderate or severe postoperative impairment of the nerve function after such treatment. ${ }^{6,17)}$ Radiosurgery is a possible treatment option for simultaneously treating AVM and neuralgia. ${ }^{5)}$ Approximately $61 \%$ to $96 \%$ of patients reportedly obtain relief of neuralgia after radiosurgery, but the recurrence rate of $\mathrm{TN}$ is approximately $14 \%$ to $23 \%$ and some time is required to achieve satisfactory pain relief. ${ }^{12,13,15)}$ If the patient requests immediate pain relief, microvascular decompression could be the first-choice of treatment. In our patient, the pain resolved immediately after microvascular decompression, and the AVM diminished following radiotherapy.

\section{References}

1) Apfelbaum RI: Surgery for tic douloureux. Clin Neurosurg 31: 351-368, 1983

2) Batjer H, Samson D: Arteriovenous malformations of the posterior fossa. Clinical presentation, diagnostic evaluation, and surgical treatment. J Neurosurg 64: 849-856, 1986

3) Dandy WE: The treatment of trigeminal neuralgia by the cerebellar route. Ann Surg 96: 787-795, 1932

4) Drake CG, Friedman AH, Peerless SJ: Posterior fossa arteriovenous malformations. J Neurosurg 64: 1-10, 1986

5) Duma CM, Lunsford LD, Kondziolka D, Bissonette DJ, Somaza S, Flickinger JC: Radiosurgery for vascular malformations of the brain stem. Acta Neurochir Suppl (Wien) 58: 92-97, 1993

6) Edwards RJ, Clarke Y, Renowden SA, Coakham HB: Trigeminal neuralgia caused by microarteriovenous malformations of the trigeminal nerve root entry zone: symptomat- ic relief following complete excision of the lesion with nerve root preservation. J Neurosurg 97: 874-880, 2002

7) Gardner WJ: Concerning the mechanism of trigeminal neuralgia and hemifacial spasm. J Neurosurg 19: 947-958, 1962

8) Ishikawa M, Nishi S, Aoki T, Takase T, Wada E, Ohwaki H, Katsuki T, Fukuda H: Operative findings in cases of trigeminal neuralgia without vascular compression: proposal of a different mechanism. J Clin Neurosci 9: 200-204, 2002

9) Jannetta PJ: Treatment of trigeminal neuralgia by suboccipital and transtentorial cranial operations. Clin Neurosurg 24: 538-549, 1977

10) Karibe $H$, Shirane R, Jokura H, Yoshimoto T: Intrinsic arteriovenous malformation of the trigeminal nerve in a patient with trigeminal neuralgia: case report. Neurosurgery 55: 1445-1449, 2004

11) Kondo A: Follow-up results of microvascular decompression in trigeminal neuralgia and hemifacial spasm. Neurosurgery 40: 46-52, 1997

12) Kondziolka D, Lunsford LD, Flickinger JC: Gamma knife radiosurgery as the first surgery for trigeminal neuralgia. Stereotact Funct Neurosurg 70 Suppl 1: 187-191, 1998

13) Maesawa S, Salame C, Flickinger JC, Pirris S, Kondziolka D, Lunsford LD: Clinical outcomes after stereotactic radiosurgery for idiopathic trigeminal neuralgia. J Neurosurg 94: 14-20, 2001

14) Maher CO, Atkinson JL, Lane JI: Arteriovenous malformation in the trigeminal nerve. Case report. J Neurosurg 98: 908-912, 2003

15) McNatt SA, Yu C, Giannotta SL, Zee CS, Apuzzo ML, Petrovich Z: Gamma knife radiosurgery for trigeminal neuralgia. Neurosurgery 56: 1295-1301, 2005

16) Mendelow AD, Erfurth A, Grossart K, Macpherson P: Do cerebral arteriovenous malformations increase in size? J Neurol Neurosurg Psychiatry 50: 980-987, 1987

17) Tsubaki S, Fukushima $T$, Tamagawa $T$, Miyazaki $S$, Watanabe K, Kuwana N, Shimizu T: Parapontine trigeminal cryptic angiomas presenting as trigeminal neuralgia. J Neurosurg 71: 368-374, 1989

Address reprint requests to: Shinya Sumioka, MD, Department of Neurosurgery, Brain, Spine and Neurology Center, Shiroyama Hospital, 2-8-1 Habikino, Habikino, Osaka 583-0872, Japan.

e-mail: s-sumioka@ energy.ocn.ne.jp 